\title{
Carbon Nanotube Based Flexible Gas Sensors Using Printing Techniques
}

\author{
Jialuo Chen ${ }^{1}$, Saswat Mishra ${ }^{2}$, Woon-Hong Yeo ${ }^{2}$, Peter J. Hesketh ${ }^{2}$, Satish Kumar ${ }^{2}$ \\ 1 School of Electrical and Computer Engineering, Georgia Institute of Technology, Atlanta USA, \\ ${ }^{2}$ G. W. Woodruff School of Mechanical Engineering, Georgia Institute of Technology, Atlanta USA, \\ satish.kumar@me.gatech.edu
}

\begin{abstract}
:
Carbon nanotube (CNT) based sensors have enormous potential in low concentration level gas detection because of its high sensitivity, fast response, and physical/chemical stability. However, device fabrication from conventional photolithography severely limit their applications when pursuing cost-effective production of flexible CNT gas sensors. In this context, printed electronic devices have attracted large interests which can provide significant design versatility and operational flexibility. Both aerosol jet and inkjet printing techniques have been used to fabricate fully printed CNT network gas sensors on flexible substrates such as polyimide (PI) and polyethylene terephthalate (PET), which have been used for ammonia and nitrogen dioxide detection in air at low ppm concentrations. To fit the corresponding sensor response, a double exponential-convolution model has been put forward. The time constants from this model are capable of capturing the different sensing phases during gas on and gas off states, which further help in deciphering the sensing mechanism. This work is of profound significance in air quality detection, pollution monitoring, and industrial emission control.
\end{abstract}

Key words: carbon nanotubes, gas sensors, flexible electronics, aerosol jet printing, inkjet printing

\section{Introduction}

Since the first CNT gas sensor was reported in 2000 by Kong et al. [1], CNT sensors have been widely studied due to their significant advantages for low power and low cost monitoring different gases at low ppm/ppb level $[2,3]$. As sensor material, CNTs have large surface area and exhibit charge-sensitive conductance. The conductance of CNTs are sensitive to ambient environment, especially to oxygen-containing gases $[4,5]$. CNT sensors can also benefit from their low cost, room temperature operation, and microelectronics applications [6]. Fabrication using printing techniques enable low-cost, high throughput manufacturing because they do not require fabrication masks or templates, which provides significant design flexibility compared to the photolithography process. As the maturity of printing techniques has developed, wearable devices have begun to emerge with outstanding flexibility and stability, such as artificial electronic skin, chemical/biological sensors, solar cells, etc.[6].

In this work, CNT gas sensors were fabricated using inkjet and aerosol jet printing techniques. Uniform CNT network on flexible substrate has been achieved through surface functionalization. Sensor response from ammonia $\left(\mathrm{NH}_{3}\right)$ and nitrogen dioxide $\left(\mathrm{NO}_{2}\right)$ spray at different concentrations were obtained in air at room temperature. A double exponential-convolution model was found to be a match to the sensing data. More importantly, the time constants from this model can well capture different sensing phases, which is used to decipher the interaction mechanism between the gas molecules and the CNT network.

\section{Fabrication}

Fig. 1(a) shows the fully printed sensors using an aerosol jet printer (Optomec AJ200). A 200 $\mu \mathrm{m}$ diameter nozzle was used for both $\mathrm{Ag}$ and CNT inks. The flow rate of sheath and carrier gas were set to $35 \mathrm{ccm}$ and $30 \mathrm{ccm}$ respectively for $\mathrm{Ag}, 75 \mathrm{ccm}$ and $45 \mathrm{ccm}$ respectively for CNT both at speed of 10 $\mathrm{mm} / \mathrm{sec}$. Oxygen plasma as pre-treatment and toluene bath as post-treatment are crucial for CNT printing. For inkjet printing, depicted in Fig. 1(b), a mesoporous PET substrate was used to achieve instant dry Ag printing (cure free, low temperature process), followed by drop casted CNT network deposition. Inkjet printing actually 
provides a rapid way for CNT sensor fabrication at extremely low cost.
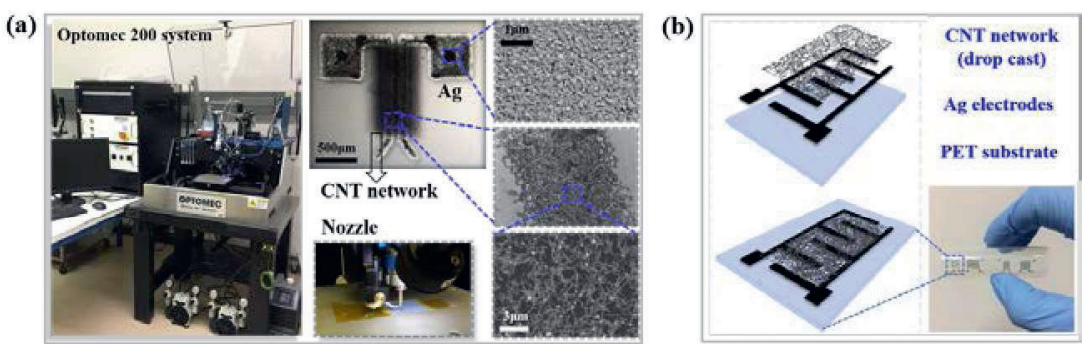

Fig.1. Printed CNT sensors. (a) Optomec aerosol jet 200 system and fully printed CNT networked sensors on a PI film. (b) CNT sensors on PET substrate using inkjet printing.
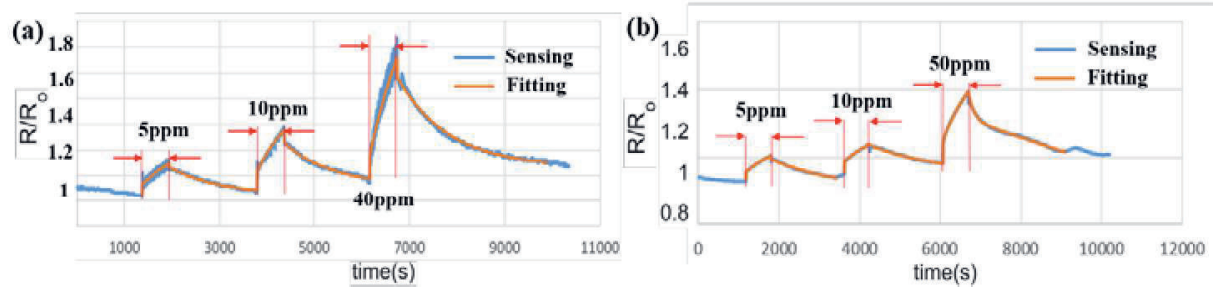

Fig.2. Sensor response and curve fitting for gas sensors fabricated using (a) aerosol jet printing and (b) inkjet printing, at room temperature $\left(25^{\circ} \mathrm{C}\right)$. The gas spray was $\mathrm{NH}_{3}$ mixed with dry $\mathrm{N}_{2}$ tested.

\section{Sensor response and modeling}

In sensor testing, gas spray was applied directly to the CNT network and Keithley 2604B was used to record the current change over time. The nozzle, with inner diameter of $5 \mathrm{~mm}$, was large enough to generate stable gas flow over the entire device. The applied gas was nitrogen $\left(\mathrm{N}_{2}\right)$ mixed with either $\mathrm{NH}_{3}$ or $\mathrm{NO}_{2}$ at different ppm concentrate level controlled by ALICAT flowmeters. The CCD camera helped probe the target device. The whole setup was located inside fume hood under atmosphere pressure and stable room temperature.

A convolution model is applied for the fitting:

$$
D_{f}(t)=f(t) \otimes g(t)
$$

Where $g(t)$ is the gas pulsing signal, and $f(t)$ is a double exponential function. The standard deviation between the fitting result $D_{f}(t)$ and sensing results $D(t)$ is defined as follows:

$$
\sigma=\left\|D_{f}(t)-D(t)\right\|=\sqrt{\frac{1}{N} \sum_{i=1}^{N}\left(D_{f}\left(t_{i}\right)-D\left(t_{i}\right)\right)^{2}}
$$

Fig. 2 shows the sensor response and fitting curves to this response under $\mathrm{NH}_{3}$ spray. Here, the fitting results are obtained from the double exponential - convolution model. This model provides a very good fit to the sensor response (with $\sigma$ as small as $\sim 10^{-4}$ ) at different gas concentrations for devices using both aerosol jet and inkjet printing. For each gas on and gas off state, two response phases at different response rates are observed (fast response rate at the beginning with sudden change of resistance, slow response rate with gradual change of resistance), which are actually characterized by two time constants obtained from the fitting by developed model. These different response phases are related to the two different response rates between gas molecules and either CNT-CNT junctions or CNT-metal junctions. The faster response corresponds to junctions or sites easily available to the gas molecule and slower response are sites for which gas molecules compete.

\section{References}

[1] J. Kong et al., "Nanotube molecular wires as chemical sensors," Science, vol. 287, no. 5453, pp. 622-625, 2000.

[2] L. Xue, W. Wang, Y. Guo, G. Liu, and P. Wan, "Flexible polyaniline/carbon nanotube nanocomposite film-based electronic gas sensors,"

Sensors and Actuators B: Chemical, vol. 244, pp. 4753, 2017.

[3] F. Rigoni et al., "Humidity-enhanced subppm sensitivity to ammonia of covalently functionalized single-wall carbon nanotube bundle layers," Nanotechnology, vol. 28, no. 255502, p. 255502, 2017.

[4] T. Someya, J. Small, P. Kim, C. Nuckolls, and J. T. Yardley, "Alcohol vapor sensors based on single-walled carbon nanotube field effect transistors," Nano letters, vol. 3, no. 7, pp. 877-881, 2003.

[5] Y. Wang et al., "Functionalized horizontally aligned CNT array and random CNT network for $\mathrm{CO}_{2}$ sensing," Carbon, vol. 117, pp. 263-270, 2017.

[6] K. Chen et al., "Printed carbon nanotube electronics and sensor systems," Advanced Materials, vol. 28, no. 22, pp. 4397-4414, 2016. 